\title{
What's Right With a Syntactic Approach to Theories and Models?
}

\author{
Sebastian Lutz
}

2010-03-31

\begin{abstract}
I argue that, contrary to common opinion, (i) unintended models do not pose a significant problem for syntactic approaches to scientific theories, (ii) in syntactic approaches, scientific theories can be as well connected to the world as in semantic ones, and (iii) some syntactic approaches are at least as language independent as semantic ones. Based on these results, I argue that syntactic and semantic approaches fare equally well when it comes to (iv) capturing the theory-observation relation, (v) ease of application, and (vi) accommodating the use of models in the sciences.
\end{abstract}

\section{Introduction}

Any analysis of a scientific theory presumes that it is captured in some way (described, shown, intuited, referred to, etc.). In this essay, I will discuss formally described theories. Suppes (1968, 654-656) lists a variety of rewards that come with formalizing a theory: explicitness, standardization, abstraction from non-essential aspects, objectivity, and the possibility of identifying self-contained, minimal assumptions. In particular, he suggests that these rewards can be reaped by using formalizations in set theory or first order predicate logic (Suppes 1968, 653), which are the most prominent examples of two greater classes that have historically been called "semantic" and "syntactic" approaches to scientific theories, respectively.

In the following, I will argue that, contrary to common opinion, a number of problems that the syntactic approaches allegedly face are solvable if they are solvable in semantic approaches. In discussing the relative merits of syntactic approaches, I will ignore the ontological question of whether theories can be identified with either kind of description (or, for that

\footnotetext{
*Theoretical Philosophy Unit, Utrecht University, The Netherlands. sebastian.lutz@gmx.net. Earlier versions of this essay were presented at at the Dutch Research Seminar for Analytic Philosophy in Utrecht and the epsa conference 2009 in Amsterdam. I thank the audiences, Thomas Müller, and Alana Yu for helpful discussions. Part of the research for this essay was undertaken during a visiting research fellowship at the Tilburg Center for Logic and Philosophy of Science.
} 
matter, with platonic objects, sets of propositions, thoughts, actions, connection weights in brains, combinations thereof...). Given that scientific theories are typically not formalized according to either kind of approach, they are probably ontologically different from both kinds of descriptions.

\section{Two ways to formalize a theory}

Syntactic approaches assume that the description of a theory by a set of at least partly interpreted sentences in predicate logic is sufficient for a wide range of philosophical analyses and definitions of important concepts like 'reduction' and 'empirical content'. Here, predicate logic is the object language, and the interpretations are given in a metalanguage.

Two remarks are in order. First, by a partial interpretation, I mean a primitive interpretation of a subset of the vocabulary or of (more generally) some expressions of the language. Any terms (elements of the vocabulary) and expressions not so interpreted receive their interpretations (if at all) through their syntactic relations to the primitively interpreted terms and expressions. A more detailed discussion is given below. Second, it is historically accurate to not restrict predicate logic to the first order. Carnap (1956) and Feigl (1970), both proponents of a specific syntactic approach known as the "received view", endorsed higher order logic for descriptions of scientific theories, as did many others.

According to Van Fraassen $(1980,44)$,

[t]he syntactic picture of a theory identifies it with a body of theorems, stated in one particular language chosen for the expression of that theory. This should be contrasted with the alternative of presenting a theory in the first instance by identifying a class of structures as its models. In this second, semantic, approach the language used to express the theory is neither basic nor unique[.]

Like Van Fraassen, Suppe (1989) and others also suggest describing a theory by identifying a class of structures, and in the following, I will focus on such semantic approaches. When relevant, I will note the relation of the discussion to the semantic approaches of Suppes (1992) and Stegmüller (1979), for example, which rely on set-theoretic formalizations.

Semantic approaches are widely thought to avoid a number of perceived shortcomings of syntactic approaches: (i) Syntactic approaches often have unintended models, unlike semantic approaches, (ii) syntactic approaches require an account of the relation between language and the world, (iii) semantic approaches are language independent, (iv) the relation between theory and observation is misleading or wrong in syntactic approaches, (v) the description of scientific theories in syntactic approaches is cumbersome, (vi) with their focus on models, semantic approaches are closer to actual scientific practice.

I will argue that if the first three of these problems can be solved for semantic approaches, they can be solved for syntactic ones as well. That the same holds for the last three problems then follows easily. 


\section{Translating between sentences and models}

It seems plausible that every syntactic description of a scientific theory can be captured by a semantic one, because any set $\Sigma$ of sentences determines the set of its models $\mathbf{A}$ through the mapping

$$
\Sigma \mapsto \mathbf{A}:=\{\mathfrak{A} \mid \mathfrak{A} \vDash \Sigma\} .
$$

This mapping loses information because it cannot distinguish between equivalent sets of sentences, that is, if $\Sigma \mapsto \mathbf{A}, \Theta \mapsto \mathbf{B}$, and $\Sigma \models \Theta$, then $\mathbf{A}=\mathbf{B}$. However, if the results of an analysis of a scientific theory are invariant under the theory's equivalent reformulation (a natural demand that is usually fulfilled), this does not pose a problem.

Conversely, any set of isomorphic structures A yields a set of sentences $\Sigma$ through the mapping

$$
\mathbf{A} \mapsto \Sigma:=\{\varphi \mid \mathfrak{A} \vDash \varphi\},
$$

which suggests that every semantic description can also be captured by a syntactic one. In general, a set of non-isomorphic structures can only be described by a set of sets of sentences. For brevity, I will restrict the following discussion to theories that do not require this. Like the previous mapping (1), the mapping (2) also loses information because it cannot distinguish between two non-identical sets of isomorphic structures, that is, if $\mathbf{A} \mapsto \Sigma, \mathbf{B} \mapsto \Theta$, and $\mathfrak{A} \simeq \mathfrak{B}$ for all $\mathfrak{A} \in \mathbf{A}, \mathfrak{B} \in \mathbf{B}$, then $\Sigma=\Theta$. However, if the results of an analysis of a scientific theory are invariant under isomorphic reformulations of the theories (also a natural demand that is usually fulfilled), this does not pose a problem either.

It is sometimes argued that more information is lost in the mapping (2) than simply the distinction between isomorphic structures. Suppe (2000, S104), for example, argues that syntactic approaches are in principle unable to capture some analyses of scientific theories, because for syntactic descriptions,

[a] general problem [is] that the Löwenheim-Skolem theorem implie[s] that [ ...] models must include both intended and wildly unintended models. Unintended models provide potential counterexamples.

Blocking them more concerns eliminating syntactical-approach artifacts than dealing with substantive analysis. [...] For example, Kitcher's (1989) unification explanation account has a very simple idea. But he develops it syntactically spending most of the paper trying to block unintended consequences that are artifacts of his formalism. [...]

This is the correct sense of [the] claim symbolic logic is an inappropriate formalism.

First and foremost, Suppe's criticism is not directed at syntactic approaches (or symbolic logic) in general, because the Löwenheim-Skolem theorem does not hold in higher order logic. And it is only in finite order logic that there are some structures that are not isomorphic but 
syntactically equivalent, that is, models of the same sentences. Furthermore, if a specific structure is given, it can typically be described up to isomorphism by a logic of some finite order. Since the proof theory of higher order logic is not complete, entailment needs to be defined semantically in terms of structures. However, there is no good reason to disallow the use of structures in syntactic approaches, since they presume only that scientific theories can be analyzed by way of their description in predicate logic. They do not presume that the analysis itself must proceed wholly in the object language. Historically, the use of structures to define entailment was certainly not anathema to Carnap $(1956,59,62)$.

Second, for predicate logic of any order, some structures can be characterized in that language up to isomorphy. If the theory has a finite domain, for example, all structures that are syntactically equivalent in a first order language are isomorphic, so that there are no unintended models.

Third, whether the existence of unintended models poses a problem depends on the kind of analysis sought after. The answers to questions that can be phrased in the object language, for example, do not depend on isomorphy, since otherwise they would provide a means of distinguishing between non-isomorphic models. And an analysis that requires isomorphy in only a finite subdomain of the theory's domain (for example in the domain of observations) is immune to the problem even in a first order language.

Finally, Suppe's criticism rests on an equivocation of 'unintended model' and 'nonstandard model', the latter referring to a model that is syntactically equivalent but not isomorphic to a standard model. Even though Kitcher spends a lot of work blocking unintended consequences, the unintended consequences are not syntactically equivalent to intended ones and can therefore be blocked by syntactic means. Kitcher's account is a good example of how difficult it can be to develop a formalization of an idea, but not of a failure of a syntactic approach because of the Löwenheim-Skolem theorem. Crudely put, if the unintended consequences could be blocked by using a predicate logic of higher order, Kitcher would probably not have shied away from it for lack of a complete proof theory.

\section{Sentences, structures, and the world}

Formally, a model $\mathfrak{A}=\langle A, f\rangle$ of a set of sentences $\Sigma$ is a domain $A$ (some set) and an interpretation $f$, a mapping from each element of the vocabulary to its extension on $A$. Now, for any such model and any set $B$ with the same cardinality as $A, B$ can be made into a model of $\Sigma$. Since $A$ has the same cardinality as $B$, there is a bijection $b: A \rightarrow B$, and $\left\langle B, b^{*} \circ f\right\rangle$ is a model of $\Sigma$, where $b^{*}$ is the mapping from possible extensions on $A$ to possible extensions on $B$ that is induced by $b$ (cf. Demopoulos and Friedman 1985). The effect is the same as that of existentially quantifying over all terms in $\Sigma$, namely, $\Sigma$ can at best determine the cardinality of the domain of its structures.

On pain of triviality, syntactic approaches must therefore assume that some terms or ex- 
pressions are primitively interpreted, that is, interpreted not only by postulating the truth of a set $\Sigma$ of sentences. Formally, a primitive interpretation restricts the set of all possible models of $\Sigma$ to a subset of so-called intended models. If the sentences are to be connected to objects in the world (e. g., animals, psychological events, or space-time areas) then some terms of the sentences have to be mapped to sets that contain at least some of these worldly objects. Let me call these sets 'worldly sets' and speak accordingly of 'worldly interpretations', 'worldly structures', and so on. Such a set of intended worldly models is, for example, assumed by Carnap (1939).

The presumption that worldly sets can be in the range of formal interpretations is somewhat problematic. To avoid it, one could simply ignore the problem of the connection to the world and assume that some sentences in $\Sigma$ are somehow primitively interpreted. Neurath (1932) was the first to suggest the use of such "protocol sentences" (cf. Carnap 1932).

Semantic approaches are sometimes claimed to avoid the problems that occur when sets of sentences are connected with the world (Chakravartty 2001, 327). How the connection to the world is to be envisaged in semantic approaches depends on whether the structures that describe the theory are worldly or non-worldly. According to Suppe $(1989,4)$, a semantic approach "construes theories as what their formulations refer to when the formulations are given a (formal) semantic interpretation", which suggests that the structures used in semantic approaches are worldly. Da Costa and French (2000, fn. 2) seem to assume the same when they note that "the set-theoretic models are constructed in set theories with Urelemente (individual[s], systems, portions of the universe, real things,...)". The connection of a set of structures to the world is in this case trivial, because the worldly objects themselves are part of the structure. While this may seem suspect, the important point is that if the description of theories by worldly structures is possible, then this immediately solves the problem of the connection between a set of sentences and the world, because these worldly structures provide an interpretation for the terms of a language.

Typically, however, the structures discussed in semantic approaches are not supposed to be worldly (e.g., French and Ladyman 1999). The structures are simply abstract set-theoretic entities-dogs, observations, or Hellhole Palms, CA, cannot be members of the sets. These non-worldly structures then have to be connected to the world. I will discuss four ways to do so, and show that each way provides a means of connecting sets of sentences to the world as much as it provides a means of connecting structures to the world.

In the first way, (some of) the structures in $\mathbf{A}$ that describe a theory are supposed to be isomorphic to some worldly structure. As in the case of the models of an uninterpreted set of sentences, any worldly set of the right cardinality can be made into a worldly structure that is isomorphic to an element of $\mathbf{A}$. Hence, if the theory is about more than just the cardinality of the domain, there has to be some specific worldly structure $\mathfrak{B}$ that is supposed to be isomorphic to (some of) the elements of $\mathbf{A}$. And then $\mathfrak{B}$ can again be used as a structure for a set of sentences. 
A second way to connect a non-worldly structure with the world is given by Muller (2010). In effect, he suggests allowing a renaming of the extensions in a structure according to expedience (more on this below). The connection to the world is given through an interpretation of the chosen vocabulary. It is clear that this approach to connect structures to the world presumes that it is possible to connect a vocabulary to the world.

In a third way, non-worldly structures represent a part of the world if they are similar to it. French and Ladyman $(1999,110 \mathrm{f})$ argue that this relation, left largely undefined, is too vague to be of much use. Be this as it may, if the similarity relation connects structures to the world, then it also connects the vocabularies interpreted by these structures to the world.

French and Ladyman $(1999,115)$ suggest that the connection to the world be left open, because this problem does not have to be solved by semantic approaches:

The theoretical models [the structures of the theory] are held to relate to models of the phenomena and these are just other structures. That these represent real events and processes cannot be determined by the content of the theory, but is a pragmatic fact about our language $[\ldots]$ and it is unreasonable to demand that the semantic view explains the nature of representation in general.

This clearly falls short of the idea that in semantic approaches, theories are easier to connect to the world than in syntactic approaches. French and Ladyman suggest considering a distinguished set of structures somehow primitively interpreted. The syntactic analogue would be a primitively interpreted set of sentences-Neurath's protocol sentences.

\section{Language independence}

According to Van Fraassen $(1980,44)$, a theory is presented by "identifying a class of structures as its models", and Suppe $(1989,4)$ states that the semantic approach "construes theories as what their formulations refer to" in a formal interpretation. In logic and model theory, structures typically involve mappings from terms to their extensions, while these quotes entail that structures consist only of extensions. Like Suppe, French and Ladyman $(1999,114 f)$ assume that the structures used in semantic approaches do not contain a vocabulary when they discuss a criticism of semantic approaches attributed to Mauricio Suárez: If a semantic approach uses models as they are defined in model theory, it is still dependent on a language, since a model "is a structure and an interpretation of a formal language in terms of that structure (that is, a map from the symbols of the syntax to elements of the structure)". If models are taken to involve such a mapping,

it is clear that the celebrated claim of the linguistic independence of considering models (and not first-order formalizations of theories), stressed by adherents of the semantic approach as giving it a clear advantage over the syntactic view, is simply not true. 
Therefore, they argue, semantic approaches should be construed as describing theories with "structures".

However, it is not exactly clear what French and Ladyman mean by 'structure', except that structures contain the extensions of terms, and not the terms themselves. They do claim that an "emphasis on structure is compatible with this definition of model theory from a contemporary textbook" (French and Ladyman 1999, 115): "Model theory is the study of the construction and classification of structures within specified classes of structures". Of course, everything in this definition by Hodges (1993, ix) depends on his definition of 'structure'. In the following quote, for example, he deals with relations (Hodges 1993, 2):

For each positive integer $n$ [a structure contains] a set of $n$-ary relations on $\operatorname{dom}(A)$ (i. e. subsets of $\left.\operatorname{dom}(A)^{n}\right)$, each of which is named by one or more $n$-ary relation symbols. If $R$ is a relation symbol, we write $R^{A}$ for the relation named by $R$.

It is clear that the terms (symbols) cannot be left out of a structure as defined by Hodges, because they identify the extensions. Let me follow Smith (2008) in calling structures so defined 'labelled structures'. Like syntactic approaches, semantic approaches using labelled structures therefore have one specific object language.

French and Ladyman would have done better with a less contemporary textbook (Bell and Slomson 1974, §3.2) in which

a relational structure is an ordered pair

$$
\mathfrak{A}=\left\langle A,\left\{R_{n}: n \in \omega\right\}\right\rangle
$$

where [...] for $n \in \omega R_{n}$ is a finitary, say $\lambda(n)$-ary relation on $A$. [...] The relational structure $\mathfrak{A}$ will count as an interpretation of the language $\mathrm{L}\left[=\left\{P_{n}\right.\right.$ : $n \in \omega\}]$ if the degrees of the relations $R_{n}$ correspond to the degrees $[\delta(n)]$ of the predicate letters $P_{n}$. That is, for $n \in \omega, \delta(n)=\lambda(n)$. In this case we say that the relational structure $\mathfrak{A}$ is a realization of the language $\mathrm{L}$ and that $\mathrm{L}$ is appropriate for the structure $\mathfrak{A}$.

This definition of 'pure structures' (Smith 2008) also seems to be used by many proponents of the semantic approach (e. g., da Costa and French 1990, French and Ladyman 1999), but it introduces a vocabulary through the back door. An indexed set $\left\{R_{n} \mid n \in \omega\right\}$ is defined through the mapping $f: \omega \rightarrow\left\{R_{n} \mid n \in \omega\right\}, n \mapsto R_{n}$, and while $\omega$ is not intended to be a vocabulary, the pure structure $\mathfrak{A}$ is formally indistinguishable from the labeled structure $\langle A, f\rangle$. So while a pure structure allows all appropriate vocabularies, $\omega$ is distinguished.

In the set-theoretic semantic approach by Suppes and Stegmüller, structures are written as tuples with the domain as first element, which can avoid this hidden dependence on a specific vocabulary. Tuples may, for example, be introduced as primitives governed by axioms or be defined through sets, as in Kuratowski's definition. Of course, if a tuple is defined as a mapping 
from (an initial sequence of) the natural numbers to a set, the result again leads to a labelled structure with the natural numbers as a vocabulary. This, incidentally, is the vocabulary that Carnap $(1958,242)$ chooses to name physical objects.

Of course, any tuple, no matter how defined, can be made into a pure structure by assigning an (initial sequence of) the natural numbers. But since this assignment is arbitrary, it is not distinguished from any other appropriate vocabulary, so that, indeed, all appropriate vocabularies are on a par. Muller (2010) has suggested making the set of all appropriate vocabularies part of a semantic approach's description of a theory. As described above, this is intended to achieve a connection between tuples and the world. My point here is that this set of appropriate vocabularies is already implicit in the definition of a tuple and a pure structure, regardless of whether it helps in connecting them to the world.

Now, like semantic approaches that rely on labelled structures, syntactic approaches are stuck with some vocabulary at first. Unlike semantic approaches that rely on tuples or, with a grain of salt, pure structures, ${ }^{1}$ syntactic approaches therefore distinguish between theories that differ only in their vocabulary. This problem of a distinction without a relevant difference can easily be solved by identifying "syntactically isomorphic" sets of sentences, which are equivalent up to a renaming of the terms (Hempel 1965, §6). But not every renaming makes sense. In the ideal gas law, for example (Suppe 1974a, 204f), the translation should allow the renaming of 'temperature' into 'températur', but not into 'pression'. This restriction on renaming can be expressed by introducing analytic sentences, in this case explicit definitions that identify one term with another. Any renaming not entailed by analytic sentences is not allowed.

The more severe problem with arbitrary renaming is that it is not only too liberal in some respects, but also to restrictive. It does not, for example, avoid the language dependence that Suppe (1974a, 204f) notes:

[Q]uantum theory can be formulated equivalently as wave mechanics or as matrix mechanics; whichever way it is formulated, it is the same theory, though its formulations as wave mechanics will constitute a collection of propositions which is different from the collection of propositions resulting from its formulations as matrix mechanics.

Obviously, the difference between matrix mechanics and wave mechanics goes beyond a mere renaming of terms. But this is a problem for the semantic view as well, because in this case, the structural description of the theory changes, too.

The use of analytic sentences can again provide a solution to this kind of language and structure dependence. So far, only the simplest kind of definition was used. In general, sets of sentences $\Sigma, \Theta$ are definitionally equivalent if and only if they have equivalent definitional

\footnotetext{
${ }^{1}$ Here, I will ignore problems for pure structures and tuples that stem from differing index sets or differing positions in the tuple, respectively.
} 
extensions. Then their models can be turned into each other: First expand the model of, say, $\Sigma$ to include the defined symbols of the definitions. This expansion is unique. Then reduce the resulting interpretation to the vocabulary of $\Theta$ (cf. Hodges 1993, 61). This allows the identification of theories that differ not only in the vocabulary they use, but also in the structures of their models. Through the mappings (2) and (1), the procedure can also be used in semantic approaches.

\section{Implications}

Syntactic approaches encounter problems with non-standard models only for very specific theories and very specific analyses, and can solve them by using an object language of higher order. Furthermore, the ways of connecting theories with the world suggested for semantic approaches can also be applied in syntactic approaches. Syntactic approaches can be as language independent as semantic approaches, and the syntactic concept of definitional equivalence can even be used to decrease the language dependence of semantic approaches.

Since the discussion leading to these results has not relied on any specific features of theories or the world, the results can be carried over to the relation between theory and observation. Arguments for the conclusion that syntactic approaches cannot describe this relation have typically relied on a specific syntactic approach, namely the received view. So while Van Fraassen $(1980,56)$ concludes very generally that the "syntactically defined relationships are simply the wrong ones", his arguments assume the received view's distinction between observational and non-observational terms. Suppes $(1967,57)$ similarly criticizes the "standard sketch" (i.e., the received view). Surprisingly, Suppe (1974a, 108, n. 225) summarizes Suppes's competing semantic approach to the theory-observation relation "in terms of theories of which [Suppes's] models are realizations", that is, as a syntactic approach.

Even if all analyses given in semantic approaches can also be given in syntactic ones, it may still be that they are more difficult in this way. Claims to this effect (e. g., Suppes 1967, 58) assume that in syntactic approaches, theories must be exhaustively described in first order logic, that is, all axioms needed for the theory, including the axioms of mathematics, have to be written out for an analysis. In contradistinction, semantic approaches allow one to silently assume the mathematics of a theory. Surely this claim is spurious. There is nothing in the use of predicate logic that forbids the silent assumption of trivial or unproblematic axioms, and indeed Carnap $(1939, \S 16)$ has done so systematically. Conversely, there is nothing in the use of set theory or model theory that forbids exhaustive axiomatization.

Finally, semantic approaches are claimed to be closer to scientific practice because of the latter's frequent use of models. However, even considering only semantic approaches that rely on models, it is doubtful that 'model' in the sciences refers to model-theoretic models, as opposed to, say, idealizations or miniatures. It is certainly possible to describe scientific models in semantic approaches, but then it is also possible to describe them in syntactic ones. 
As far as the six alleged advantages of semantic approaches are concerned, I can now say what is right with syntactic approaches: everything that is right with semantic ones (and possibly more).

\section{References}

Bell, J. L. and Slomson, A. B. (1974). Models and Ultradproducts: An Introduction. NorthHolland, Amsterdam, $3^{\text {rd }}$ edition.

Carnap, R. (1932). Über Protokollsätze. Erkenntnis, 3(1):215-228.

Carnap, R. (1939). Foundations of Logic and Mathematics, volume I,3 of Foundations of the Unity of Science. University of Chicago Press, Chicago. Page numbers refer to the two volume edition.

Carnap, R. (1956). The methodological character of theoretical concepts. In Feigl, H. and Scriven, M., editors, The Foundations of Science and the Concepts of Psychology and Psychoanalysis, volume 1 of Minnesota Studies in the Philosophy of Science. University of Minnesota Press, Minneapolis.

Carnap, R. (1958). Beobachtungssprache und theoretische Sprache. Dialectica, 12:236-248.

Chakravartty, A. (2001). The semantic or model-theoretic view of theories and scientific realism. Synthese, 127:325-345.

da Costa, N. and French, S. (1990). The model-theoretic approach in the philosophy of science. Philosophy of Science, 57:248-265.

da Costa, N. and French, S. (2000). Models, theories, and structures: Thirty years on. Philosophy of Science, 67:S116-S127.

Demopoulos, W. and Friedman, M. (1985). Bertrand Russell's The Analysis of Matter: Its historical context and contemporary interest. Philosophy of Science, 52(4):621-639.

Feigl, H. (1970). The "orthodox" view of theories: Remarks in defense as well as critique. In Radner, M. and Winokur, S., editors, Analyses of Theories and Methods of Physics and Psychology, volume 4 of Minnesota Studies in the Philosophy of Science, pages 3-16. University of Minnesota Press, Minneapolis. 
French, S. and Ladyman, J. (1999). Reinflating the semantic approach. International Studies in the Philosophy of Science, 13(2):103-121.

Hempel, C. G. (1965). Aspects of scientific explanation. In Aspects of Scientific Explanation and Other Essays in the Philosophy of Science, pages 331-496. The Free Press, New York.

Hodges, W. (1993). Model Theory. Cambridge University Press, Cambridge.

Kitcher, P. and Salmon, W., editors (1989). Scientific Explanation, volume 13 of Minnesota Studies in the Philosophy of Science. University of Minnesota Press, Minneapolis.

Muller, F. A. (2010). Reflections on the revolution at Stanford. Synthese.

Neurath, O. (1932). Protokollsätze. Erkenntnis, 3(1):204-214.

Smith, P. (2008). Introducing Wilfrid Hodges, A Shorter Model Theory. Typescript.

Stegmüller, W. (1979). The Structuralist View of Theories. Springer Verlag, New York.

Suppe, F. (1974a). The search for philosophic understanding of scientific theories. In Suppe (1974b), pages 3-241.

Suppe, F., editor (1974b). The Structure of Scientific Theories. University of Illinois Press, Urbana.

Suppe, F. (1989). The Semantic Conception of Theories and Scientific Realism. University of Illinois Press, Urbana.

Suppe, F. (2000). Understanding scientific theories: An assessment of developments, 19691998. Philosophy of Science, 67:S102-S115.

Suppes, P. (1967). What is a scientific theory? In Morgenbesser, S., editor, Philosophy of Science Today, pages 55-67. Basic Books, New York.

Suppes, P. (1968). The desirability of formalization in science. The Journal of Philosophy, 65(20):651-664.

Suppes, P. (1992). Axiomatic methods in science. In Carvallo, M. E., editor, Nature, Cognition and System II, pages 205-232. Springer, Heidelberg.

van Fraassen, B. C. (1980). The Scientific Image. Clarendon Press, Oxford. 